\title{
On automorphisms of the punctual Hilbert schemes of K3 surfaces
}

\author{
Keiji Oguiso ${ }^{1,2}$
}

Received: 25 December 2014 / Revised: 5 August 2015 / Accepted: 8 August 2015 /

Published online: 19 November 2015

(C) Springer International Publishing AG 2015

\begin{abstract}
We present a sufficient condition for the punctural Hilbert scheme of length two of a K3 surface with finite automorphism group to have automorphism group of infinite order in geometric terms (Theorem 2.1) and supply it with concrete examples (Theorem 1.2). We also discuss Mori dream space structures under an extermal crepant resolution (Theorems 1.2, 4.1, 5.2) from the viewpoint of automorphisms. These affirmatively answer a question of Malte Wandel.
\end{abstract}

Keywords Hyperkähler manifolds · Automorphisms · Mori dream space $\cdot$ Crepant resolution

Mathematics Subject Classification $\quad 14 J 50 \cdot 14 J 35 \cdot 14 J 28$

\section{Introduction}

Throughout this note, we work over the complex number field $\mathbb{C}$. We call a $\mathbb{C}$-valued point $P$ of a variety $V$ simply a point and write $P \in V$ instead of $P \in V(\mathbb{C})$. Topology of a complex variety is assumed to be the Euclidean topology of the underlying analytic

Dedicated to Professor Doctor Fabrizio Catanese on the occasion of his sixty-fifth birthday.

The author is supported by JSPS Grant-in-Aid (S) 25220701, JSPS Grant-in-Aid (S) 15H05738, JSPS Grant-in-Aid (B) 15H03611, and by KIAS Scholar Program.

$凶 \quad$ Keiji Oguiso

oguiso@math.sci.osaka-u.ac.jp

1 Department of Mathematics, Osaka University, Toyonaka, Osaka 560-0043, Japan

2 Korea Institute for Advanced Study, Hoegiro 87, Seoul 133-722, Korea 
space. We denote the group of biregular automorphisms of $V$ by $\operatorname{Aut}(V)$ and the group of birational automorphisms of $V$ by $\operatorname{Bir}(V)$.

Let $S$ be a smooth projective surface. We denote by $S^{[n]}$ the Hilbert scheme Hilb ${ }^{n}(S)$ of 0-dimensional closed subschemes of length $n \geq 2$ on $S$ and by $S^{(n)}$ the Chow variety $S^{n} / \Sigma_{n}$ of 0-dimensional cycles of length $n \geq 2$ on $S$. Here $S^{n}$ is the $n$-times self-product of $S$ and $\Sigma_{n}$ is the symmetric group of $n$-letters which acts on $S^{n}$ by $\left(P_{i}\right)_{i=1}^{n} \mapsto\left(P_{\sigma(i)}\right)_{i=1}^{n}$.

Let $S$ be a projective K3 surface, i.e., a smooth, simply-connected projective surface $S$ with $\mathcal{O}_{S}\left(K_{S}\right) \simeq \mathcal{O}_{S}$. Here $K_{S}$ is a canonical divisor of $S$. Then $S^{[n]}$ is a projective hyperkähler manifold, i.e., a smooth, simply-connected projective variety with everywhere non-degenerate global regular 2-form $\sigma_{S^{[n]}}$ such that $H^{0}\left(S^{[n]}, \Omega_{S^{[n]}}^{2}\right)=$ $\mathbb{C} \sigma_{S^{[n]}}[5,8]$.

The aim of this note is to give an affirmative answer to the following question asked by Malte Wandel (Theorem 1.2, see also Theorems 2.1, 4.1, 5.2).

Question 1.1 Is it possible that $\left|\operatorname{Aut}\left(S^{[2]}\right)\right|=\infty$ for a projective K3 surface $S$ with $|\operatorname{Aut}(S)|<\infty$ ?

Note that $\operatorname{Aut}(S)$ naturally and faithfully acts on $S^{[n]}$. Hence $\left|\operatorname{Aut}\left(S^{[n]}\right)\right|=\infty$ if $|\operatorname{Aut}(S)|=\infty$.

Throughout this note, we denote by $\Lambda$ the even hyperbolic lattice of rank 2 and of discriminant 17 , defined by

$$
\Lambda=\left(\mathbb{Z}^{2}=\mathbb{Z} e_{1}+\mathbb{Z} e_{2}:\left(\left(e_{i}, e_{j}\right)\right)=\left(\begin{array}{ll}
2 & 5 \\
5 & 4
\end{array}\right)\right), \quad\left|\operatorname{det}\left(\begin{array}{ll}
2 & 5 \\
5 & 4
\end{array}\right)\right|=17
$$

Here, by a lattice $L=(L,(\cdot, \cdot))$, we mean a pair of a free $\mathbb{Z}$-module $L$ of finite rank $r$ and a non-degenerate $\mathbb{Z}$-valued symmetric bilinear form $(\cdot, \cdot)$ on $L$. The lattice $L$ is called hyperbolic if $(\cdot, \cdot) \otimes \mathbb{R}$ is of signature $(1, r-1)$, and even if $(x, x) \in 2 \mathbb{Z}$ for all $x \in L$. Lattice isomorphisms are defined in an obvious manner. The Néron-Severi group NS $(S)$ of a projective K3 surface $S$ is an even hyperbolic lattice with respect to the intersection paring $(\cdot, \cdot)$ (see e.g. [3, Chapter VIII]). Its rank, the Picard number of $S$, is denoted by $\rho(S)$.

Our main result is the following.

Theorem 1.2 Let $S$ be a (necessarily projective) K3 surface such that $\mathrm{NS}(S) \simeq \Lambda$. Then:

(a) $\left|\operatorname{Aut}\left(S^{[2]}\right)\right|=\infty$ and $|\operatorname{Aut}(S)|<\infty$.

(b) The Hilbert-Chow morphism $\mu: S^{[2]} \rightarrow S^{(2)}$ is an extremal crepant resolution such that the source $S^{[2]}$ is not a Mori dream space, but the target $S^{(2)}$ is a Mori dream space.

See also Theorem 2.1 in Sect. 2 for a geometric criterion related to Theorem 1.2 (a). The essential point of the proof of Theorem 1.2(a) is as follows: $S$ in Theorem 1.2 has at least two different embeddings into $\mathbb{P}^{3}$. The images are smooth quartic surfaces with no line. From this, we see that $S^{[2]}$ has at least two different biregular involutions, called Beauville's involutions [4, Section 6], corresponding to these two embeddings. See Sects. 2 and 3 for details. 
The notion of Mori dream space (MDS) was introduced in [10, Definition 11]. See also Definition 5.1 in Sect. 5. Theorem 1.2(b) was kindly suggested to me by Shinnosuke Okawa. See also Theorems 4.1 and 5.2 in Sects. 4 and 5 for slightly more general results related to Theorem 1.2(b).

Remark 1.3 K3 surfaces $S$ in Theorem 1.2 form a dense subset of the 18-dimensional family of $\Lambda$-polarized K3 surfaces ([7], see also [17, Corollary 2.9] for the existence).

Remark 1.4 (a) If $S$ is a K3 surface with $\rho(S)=1$, then both $\operatorname{Aut}\left(S^{[n]}\right)$ and $\operatorname{Bir}\left(S^{[n]}\right)$ are finite groups (see e.g. [19, Corollary 5.2]). By the definition, $\rho(S)=2$ for K3 surfaces $S$ in Theorem 1.2. Thus, $\rho(S)=2$ is the smallest Picard number for Question 1.1 to have an affirmative answer.

(b) Theorem 2.1 in Sect. 2 and our proof of Theorem 1.2 (a) in Sect. 3 suggest that there will be many other ways to construct examples similar to the ones in Theorem 1.2. Compare with [15, Section 3].

We close the introduction by recalling the following question of Alessandra Sarti.

Question 1.5 Is $\operatorname{Aut}\left(X^{[n]}\right)=\operatorname{Aut}(X)$ for any projective K3 surface $X$ and any $n \geq 3$ ?

Remark 1.6 If Question 1.5 would have an affirmative answer for our $S$ in Theorem 1.2, then $\operatorname{Aut}\left(S^{[n]}\right)=\infty$ only when $n=2$. A work of Markman and Yoshioka [16] might be of some help to check this. Brendan Hassett also kindly informed me that he found a counterexample for Question 1.5 in $n=3$.

\section{A geometric criterion}

In this section, we shall prove the following Theorem 2.1. In fact, our proof of Theorem 1.2 (a) will be reduced to this theorem in Sect. 3.

Theorem 2.1 Let $S$ be a projective K3 surface of Picard number $\rho(S)=2$ such that:

(a) S contains either a smooth elliptic curve or a smooth rational curve;

(b) Shas very ample divisors $H_{1}, H_{2}$ such that

$$
\left(H_{1}, H_{1}\right)=\left(H_{2}, H_{2}\right)=4, \quad H_{1} \neq H_{2},
$$

in $\mathrm{NS}(S)$; and

(c) S has no smooth rational curve $C_{k}$ such that $\left(C_{k}, H_{k}\right)=1$ for each $k=1,2$.

Then $|\operatorname{Aut}(S)|<\infty$ and $\left|\operatorname{Aut}\left(S^{[2]}\right)\right|=\infty$.

Proof By (a), the nef cone $\overline{\operatorname{Amp}}(S) \subset \mathrm{NS}(S) \otimes \mathbb{R}$ has at least one boundary ray defined over $\mathbb{Q}$, i.e., the class of the elliptic curve or the orthogonal to the smooth rational curve with respect to the intersection pairing. Since $\rho(S)=2$, this implies $|\operatorname{Aut}(S)|<\infty$ by [19, Proposition 2.4].

By (b), we have two embeddings

$$
\Phi_{k}=\Phi_{\left|H_{k}\right|}: S \rightarrow \mathbb{P}^{3}
$$


associated to the complete linear systems $\left|H_{k}\right|, k=1,2$. Let $S_{k}=\Phi_{k}(S)$. Then $S_{k} \subset \mathbb{P}^{3}$ is a smooth quartic surface by (b) and $S_{k}$ contains no line by (c).

Let $P, Q \in S$ be general points of $S$. Then, the projective line $\Phi_{k}(P) \Phi_{k}(Q)$ meets $S_{k}$ in four points, namely, $\Phi_{k}(P), \Phi_{k}(Q), \Phi_{k}(R), \Phi_{k}(T)$. The correspondence $\{P, Q\} \mapsto\{R, T\}$ then defines a birational automorphism of $S^{[2]}$ of order 2 for each $k=1,2$,

$$
\iota_{k}: S^{[2]} \rightarrow S^{[2]}
$$

$\iota_{k}$ is called the Beauville involution with respect to $H_{k}$ [4, Proposition 11]. As $S_{k} \subset \mathbb{P}^{3}$ contains no projective line, $\iota_{k}$ is biregular, i.e., $\iota_{k} \in \operatorname{Aut}\left(S^{[2]}\right)$ [4, Proposition 11].

Recall that NS $\left(S^{[2]}\right)$ is a hyperbolic lattice of rank $3=\rho(S)+1$ with respect to the Beauville-Bogomolov form [5, Proposition 6]. More strongly, we have the following natural identification as lattices [5, Proposition 6]:

$$
\mathrm{NS}\left(S^{[2]}\right)=\mathrm{NS}(S) \oplus \mathbb{Z} e, \quad(e, e)=-2 .
$$

Here $e=E / 2$ for the exceptional divisor $E$ of the Hilbert-Chow morphism $v: S^{[2]} \rightarrow$ $S^{(2)}$ [5, Proposition 6]. Note that $H_{1}$ and $H_{2}$ are linearly independent in $\mathrm{NS}(S)$. Hence we have

$$
\mathrm{NS}\left(S^{[2]}\right) \otimes \mathbb{Q}=\mathbb{Q}\left\langle H_{1}-e, H_{2}-e, e\right\rangle .
$$

Consider the action of the Beauville involution $i_{k}$ on $\mathrm{NS}\left(S^{[2]}\right)$,

$$
\iota_{k}^{*}: \mathrm{NS}\left(S^{[2]}\right) \rightarrow \mathrm{NS}\left(S^{[2]}\right)
$$

This is the anti-involution with respect to the invariant vector $H_{k}-e$,

$$
\iota_{k}^{*}(x)=-x+\left(x, H_{k}-e\right)\left(H_{k}-e\right)
$$

Here we note that $\left(H_{k}-e, H_{k}-e\right)=2$. This formula is due to Debarre [6, Theorem 4.1], see also [18, Proposition 4.1]. Then, with respect to the $\mathbb{Q}$-basis $\left\langle H_{1}-e, H_{2}-e, e\right\rangle$ of $\mathrm{NS}\left(S^{[2]}\right) \otimes \mathbb{Q}$, we have the following matrix representations of $\iota_{k}^{*}$ :

$$
\iota_{1}^{*}=\left(\begin{array}{ccc}
1 & m-2 & 2 \\
0 & -1 & 0 \\
0 & 0 & -1
\end{array}\right), \quad \iota_{2}^{*}=\left(\begin{array}{ccc}
-1 & 0 & 0 \\
m-2 & 1 & 2 \\
0 & 0 & -1
\end{array}\right)
$$

Here we put $m=\left(H_{1}, H_{2}\right)$. As $m$ is a positive integer and

$$
m^{2}=\left(H_{1}, H_{2}\right)^{2}>\left(H_{1}, H_{1}\right)\left(H_{2}, H_{2}\right)=4^{2}
$$


by the Hodge index theorem, it follows that $m \geq 5$. Observe then that

$$
\left(\iota_{2} \iota_{1}\right)^{*}=\iota_{1}^{*} \iota_{2}^{*}=\left(\begin{array}{ccc}
(m-2)^{2}-1 & m-2 & 2 m-6 \\
-(m-2) & -1 & -2 \\
0 & 0 & 1
\end{array}\right) .
$$

Let $F(t)$ be the characteristic polynomial of $\left(\iota_{2} \iota_{1}\right)^{*}$. Then

$$
F(t)=f(t)(t-1), \quad f(t)=t^{2}-\left((m-2)^{2}-2\right) t+1 .
$$

By $m \geq 5$, we have

$$
(m-2)^{2}-2 \geq(5-2)^{2}-2 \geq 7, \quad D=\left((m-2)^{2}-2\right)^{2}-4>0 .
$$

Hence the quadratic polynomial $f(t)$ has two distinct real roots. We denote them by $\alpha<\beta$. As

$$
2 \beta>\alpha+\beta=(m-2)^{2}-2 \geq 7,
$$

it follows that $\beta>1$. Let $n$ be an integer. Then $\beta^{n}$ is an eigenvalue of $\left(\left(\iota_{2} \iota_{1}\right)^{*}\right)^{n}$. As $\beta>1$, it follows that $\beta^{n}=1$ implies that $n=0$. Thus $\left(\iota_{2} \iota_{1}\right)^{*}$ is of infinite order. Hence $\iota_{2} \iota_{1} \in \operatorname{Aut}\left(S^{[2]}\right)$ is of infinite order as well. This completes the proof of Theorem 2.1.

\section{Proof of Theorem 1.2(a)}

In this section, we shall prove Theorem 1.2(a). We proceed by dividing into several steps. Set

$$
\mathrm{NS}(S)=\mathbb{Z}\langle L, H\rangle, \quad(L, L)=2, \quad(H, H)=4, \quad(L, H)=5 .
$$

Claim 3.1 There is no $C \in \mathrm{NS}(S) \backslash\{0\}$ such that $(C, C)=0$. In particular, $S$ has no curve $C$ with arithmetic genus $p_{a}(C)=1$.

Proof Write $C=x L+y H$ in $\operatorname{NS}(S)$. Here $x, y \in \mathbb{Z}$. Then

$$
(C, C)=2 x^{2}+10 x y+4 y^{2} .
$$

So, if $(C, C)=0$, then $x=(-5 \pm \sqrt{17}) y / 2$. As $x, y \in \mathbb{Z}$ and $(-5 \pm \sqrt{17}) / 2 \notin \mathbb{Q}$, it follows that $y=0$ and therefore $x=0$. Hence $C=0$ in $\operatorname{NS}(S)$.

Claim 3.2 There is no $C \in \mathrm{NS}(S)$ such that $(L, C)=0$ and $(C, C)=-2$. In particular, there is no smooth rational curve $C \subset S$ such that $(L, C)=0$. 
Proof Write $C=x L+y H$ in $\operatorname{NS}(S)$. Here $x, y \in \mathbb{Z}$. Then

$$
(L, C)=2 x+5 y \text {. }
$$

If $(L, C)=0$, then there is $z \in \mathbb{Z}$ such that $x=5 z$ and $y=-2 z$. Then

$$
(C, C)=(5 L-2 H, 5 L-2 H) z^{2}=-34 z^{2} \neq-2 .
$$

This implies the first assertion of Claim 3.2. For the last assertion, we note that if $C$ is a smooth rational curve on $S$, then $(C, C)=-2$ by the adjunction formula.

Set $P(S)=\{x \in \mathrm{NS}(S) \otimes \mathbb{R}:(x, x)>0\}$ and denote by $P^{+}(S)$ the $\mathbb{Q}$-rational hull of $P(S)$ in $\mathrm{NS}(S) \otimes \mathbb{R}$, i.e., the union of $P(S)$ and $\mathbb{Q}$-rational points on the boundary $\partial P(S)$ with respect to the integral structure of NS $(S)$. Let $\operatorname{Amp}(S) \subset \mathrm{NS}(S) \otimes \mathbb{R}$ be the ample cone of $S$ and $\operatorname{Amp}^{+}(S)$ be the $\mathbb{Q}$-rational hull of $\operatorname{Amp}(S)$ in $\mathrm{NS}(S) \otimes \mathbb{R}$. Note that $\operatorname{Amp}^{+}(S) \subset P^{+}(S)$. Let $W(\mathrm{NS}(S))$ be the group generated by the reflections on $\mathrm{NS}(S)$ :

$$
r_{C}: x \mapsto x+(x, C) C
$$

Here $C$ runs through all the elements of $\operatorname{NS}(S)$ such that $(C, C)=-2$. The product group $W(\mathrm{NS}(S)) \times\left\{ \pm \operatorname{id}_{\mathrm{NS}(S)}\right\}$ acts on both $P^{+}(S)$ and $P^{+}(S) \cap \mathrm{NS}(S)$.

Claim 3.3 $\mathrm{Amp}^{+}(S)$ is the fundamental domain of the action of $W(\mathrm{NS}(S))$ $\times\left\{ \pm \operatorname{id}_{\mathrm{NS}(S)}\right\}$ on $P^{+}(S)$.

Proof This is a version of the Nakai-Moishezon criterion for the ampleness of line bundles on a projective K3 surface, see e.g., [3, Chapter VIII].

By Claim 3.3, we may and will assume that $L \in \operatorname{Amp}^{+}(S) \cap \operatorname{NS}(S)$. Then $L$ is nef and big. More strongly, we have

Claim 3.4 L is ample.

Proof Since $L$ is nef and big, $L=K_{S}+L$ is semi-ample by the base point free theorem [11]. So, if $L$ were not ample, then there were an irreducible curve $C \subset S$ such that $(L, C)=0$. By the Hodge index theorem, $(C, C)<0$. However, then $p_{a}(C)=0$ by the adjunction formula, and $C$ were a smooth rational curve on $S$ with $(L, C)=0$, a contradiction to Claim 3.2.

From now on, we assume that $L \in \mathrm{NS}(S) \simeq \operatorname{Pic}(S)$ is an ample class with $(L, L)=2$.

Claim 3.5 The complete linear system $|L|$ is free and the associated morphism

$$
\Phi=\Phi_{|L|}: S \rightarrow \mathbb{P}^{2}
$$

is a finite double cover. 
Proof We prove first that the complete linear system $|L|$ is free. Consider the decomposition

$$
|L|=|M|+F .
$$

Here $|M|$ is the movable part and $F$ is the fixed component of $|L|$. If $|L|$ would not be free, then $F$ would be effective and non-zero by [21, Corollary 3.2]. Then

$$
2=(L, L)>(L, M) \geq(M, M) \geq 0 .
$$

As $(M, M) \in 2 \mathbb{Z}$, it would follow that $(M, M)=0$, a contradiction to Claim 3.1. Hence $|L|$ is free. As $(L, L)=2$ and $L$ is ample, we have $h^{0}(S, L)=3$ by the Riemann-Roch formula. Hence $\Phi_{|L|}$ is a finite double covering of $\mathbb{P}^{2}$ as claimed.

Let $\tau \in \operatorname{Bir}(S)$ be the covering involution of $\Phi: S \rightarrow \mathbb{P}^{2}$.

Claim 3.6 $\tau \in \operatorname{Aut}(S)$ and

$$
\tau^{*}=\left(\begin{array}{cc}
1 & 5 \\
0 & -1
\end{array}\right)
$$

on $\mathrm{NS}(S)$ with respect to the $\mathbb{Z}$-basis $\langle L, H\rangle$ of $\mathrm{NS}(S)$.

Proof Note that $\operatorname{Bir}(S)=\operatorname{Aut}(S)$ as $S$ is a smooth projective surface with nef $K_{S}$. Hence $\tau \in \operatorname{Aut}(S)$. By the definition, $L=\Phi^{*} \mathcal{O}_{\mathbf{P}^{2}}(1)$. Thus $\tau^{*} L=L$.

We have

$$
\Phi^{*} \Phi_{*} H=H+\tau^{*} H
$$

Here $\Phi_{*} H$ is the pushforward as 1 -cycles. Then $\Phi_{*} H \in\left|\mathcal{O}_{\mathbb{P}^{2}}(k)\right|$ for some $k \in \mathbb{Z}_{>0}$. Then, by the projection formula, we have

$$
\left(\Phi^{*} \Phi_{*} H, L\right)=\left(\Phi^{*} \Phi_{*} H, \Phi^{*} \mathcal{O}_{\mathbb{P}^{2}}(1)\right)=2\left(\Phi_{*} H, \mathcal{O}_{\mathbb{P}^{2}}(1)\right)=2 k
$$

On the other hand, as $\Phi^{*} \Phi_{*} H=H+\tau^{*} H$ and $\tau^{*} L=L$, we also have

$$
\begin{aligned}
\left(\Phi^{*} \Phi_{*} H, L\right) & =\left(H+\tau^{*} H, L\right)=(H, L)+\left(\tau^{*} H, L\right) \\
& =(H, L)+\left(\tau^{*} H, \tau^{*} L\right)=2(H, L)=10 .
\end{aligned}
$$

Hence $k=5$ and $H+\tau^{*} H=\Phi^{*}\left(\mathcal{O}_{\mathbb{P}^{2}}(5)\right)$. As $L=\Phi^{*} \mathcal{O}_{\mathbf{P}^{2}}(1)$, it follows that $\tau^{*} H=5 L-H$. Combining this with $\tau^{*} L=L$, we obtain the last assertion.

Claim 3.7 If $C$ is an irreducible curve on $S$ with $(C, C)<0$, then $C$ is a smooth rational curve and $|C|=\{C\}$.

Proof This is well-known and easily follows from the irreducibility of $C$ and the adjunction formula, see e.g. [3, Chapter VIII]. 
Claim 3.8 (a) S has exactly two smooth rational curves and their classes in $\operatorname{NS}(S)$ are

$$
-L+2 H, \quad \tau^{*}(-L+2 H)=9 L-2 H .
$$

(b) The cone of effective curves on $S$ is

$$
\overline{\mathrm{NE}}(S)=\mathbb{R}_{\geq 0}(-L+2 H)+\mathbb{R}_{\geq 0}(9 L-2 H) .
$$

(c) S satisfies condition (a) in Theorem 2.1.

Proof Assertion (c) follows from (b). Recall that $\rho(S)=2$. Then (b) follows from (a) and Claim 3.7. The fact that $\tau^{*}(-L+2 H)=9 L-H$ follows from Claim 3.6. Set

$$
C_{1}=-L+2 H, \quad C_{2}=9 L-2 H .
$$

Then $\left(C_{i}, C_{i}\right)=-2$ and $\left(C_{i}, L\right)=8$ for each $i=1,2$. Moreover, the classes $C_{i}$ are integral and primitive, i.e., indivisible in $\mathrm{NS}(S)$. Hence, if we have an irreducible curve $D$ with negative self intersection, the class of $D$ is either $C_{1}$ or $C_{2}$. Here we also used the fact that $\rho(S)=2$. By the Riemann-Roch formula, $C_{1}$ and $C_{2}$ are represented by effective curves, say, $D_{1}$ and $D_{2}$ with $\left(D_{i}, D_{i}\right)=-2<0$. If $D_{i}$ is not irreducible, then $D_{i}$ contains an irreducible curve $D$ of negative self intersection and we have an equality of effective divisors $D_{i}=D+E$ for some effective curve $E \neq 0$. Since $D$ is an irreducible curve of negative self intersection, the class of $D$ is either $C_{1}$ or $C_{2}$. However, then

$$
(E, L)=\left(C_{i}, L\right)-\left(C_{j}, L\right)=8-8=0,
$$

a contradiction to the fact that $E \neq 0$ is effective and $L$ is ample. Hence both $D_{i}$ are irreducible. Combining this with Claim 3.7, we obtain (a).

Now, we shall show that $H$ and $\tau^{*} H$ satisfy the conditions (b) and (c) in Theorem 2.1. We proceed by dividing into several steps.

Claim 3.9 $H$ is ample and $h^{0}(S, H)=4$.

Proof Observe that

$$
(H,-L+2 H)=3>0, \quad(H, 9 L-2 H)=37>0 .
$$

Thus $H$ is ample by the Kleiman's criterion and Claim $3.8(\mathrm{~b})$. As $(H, H)=4$, the second assertion now follows from the Riemann-Roch formula.

Claim 3.10 $|H|$ has no fixed component. 
Proof Assume to the contrary that $|H|$ has a fixed component. Then $|H|=|M|+F$, where $|M|$ is the movable part and $F$ is a non-zero effective divisor. Using Claim 3.1, we have

$$
4=(H, H)>(H, M) \geq(M, M)>0, \quad 5=(H, L)>(M, L)>0 .
$$

Therefore $(M, M)=2$ by Claim 3.1 and by the fact that $(M, M) \in 2 \mathbb{Z}$. Put $k=$ $(M, L)$. Then $1 \leq k \leq 4$ by the second inequality above, and the discriminant of $\mathbb{Z}\langle L, M\rangle$ is $\left|k^{2}-4\right|$, possibly 0 . For the same reason as in Claim 3.8, we have

$$
\left|k^{2}-4\right|=17 l^{2}
$$

for some $l \in \mathbb{Z}$. As $l$ and $k$ are integers such that $1 \leq k \leq 4$, it follows that $k=2$ and therefore $\left|k^{2}-4\right|=0$. Thus $M=m L$ for some $m \in \mathbb{Q}$ by the Hodge index theorem. Then, by $(M, M)=2=(L, L)$, it follows that $M=L$ in $\operatorname{Pic}(S) \simeq \operatorname{NS}(S)$, a contradiction to the fact that $h^{0}(S, M)=h^{0}(S, H)=4$ and $h^{0}(S, L)=3$.

Claim 3.11 $H$ is very ample.

Proof By [21, Theorem 5.2] and by Claim 3.10, it suffices to check the following two facts:

(i) There is no irreducible curve $E \subset S$ such that $p_{a}(C)=1$ and $(E, H)=2$.

(ii) There is no irreducible curve $B \subset S$ such that $p_{a}(B)=2$ and $H=2 B$ in $\operatorname{NS}(S)$.

The assertion (i) follows from Claim 3.1. By the adjunction formula, $(B, B)=2$ if $p_{a}(B)=2$. The assertion (ii) follows from this equality and $(H, H)=4$.

Claim 3.12 There is no smooth rational curve $C$ on $S$ such that $(H, C)=1$.

Proof Recall that the class of $C$ is either $-L+2 H$ or $\tau^{*}(-L+2 H)=9 L-2 H$ by Claim 3.8. The result now follows from

$$
(H,-L+2 H)=3 \neq 1, \quad(H, 9 L-2 H)=37 \neq 1 .
$$

Put $H_{1}=H$ and $H_{2}=\tau^{*} H$. Then $H_{2}=5 L-H$ by Claim 3.6.

Claim 3.13 $H_{1}$ and $H_{2}$ satisfy conditions (b) and (c) in Theorem 2.1.

Proof As $H_{1} \neq H_{2}$ in $\mathrm{NS}(S),(H, H)=4$ and $\tau \in \operatorname{Aut}(S)$, the result follows from Claims 3.11 and 3.12.

Theorem 1.2(a) now follows from Theorem 2.1 and Claims 3.8 and 3.13.

\section{Some properties of the product of $\mathrm{K} 3$ surfaces}

Let $V$ be a normal $\mathbb{Q}$-factorial projective variety. We denote by $\omega_{V}$ the dualizing sheaf of $V$. A divisor $D$ is called movable if there is a positive integer $k$ such that the complete linear system $|k D|$ has no fixed component. A divisor class $d \in \mathrm{NS}(V)$ is 
called movable if $d$ is represented by a movable divisor $D$. By $\operatorname{Mov}(V) \subset \mathrm{NS}(V) \otimes \mathbb{R}$, we denote the movable cone of $M$, i.e., the convex cone generated by the movable divisor classes. We denote by $\overline{\operatorname{Mov}}(V)$ the closure of the movable cone in the linear space $\mathrm{NS}(V) \otimes \mathbb{R}$.

By $\operatorname{Amp}(V) \subset \mathrm{NS}(V) \otimes \mathbb{R}$, we denote the ample cone of $V$, i.e., the open convex cone generated by the ample divisor classes. We denote by $\overline{\operatorname{Amp}}(V)$ the closure of the ample cone in $\mathrm{NS}(V) \otimes \mathbb{R}$. The cone $\overline{\operatorname{Amp}}(V)$ is called the nef cone.

Let $n$ be a positive integer, possibly $n=1$, and $S_{i}, 1 \leq i \leq n$, be mutually nonisomorphic projective $\mathrm{K} 3$ surfaces. Let $m_{i} \geq 1,1 \leq i \leq n$, be positive integers (not necessarily distinct). We consider the following product:

$$
M=S_{1}^{m_{1}} \times S_{2}^{m_{2}} \times \cdots \times S_{n}^{m_{n}}
$$

$M$ is a smooth projective variety. We denote the tangent bundle of $M$ by $T_{M}$. We denote the $(i, j)$-th factor of $M$ by $S_{i j}$ and by

$$
p_{i j}: M \rightarrow S_{i j}
$$

the projection to the $(i, j)$-th factor. Here $1 \leq i \leq n, 1 \leq j \leq m_{i}$. Note that $S_{i j}=S_{i}$ as abstract varieties. We identify

$$
\mathrm{NS}\left(S_{i j}\right)=p_{i j}^{*} \mathrm{NS}\left(S_{i j}\right) \subset \mathrm{NS}(M)
$$

We denote by $\Sigma_{k}$ the symmetric group of $k$-letters. Then the group $\operatorname{Aut}\left(S_{i}\right)^{m_{i}} \rtimes \Sigma_{m_{i}}$ naturally acts on $S_{i}^{m_{i}}$ and hence on $M$. More precisely, by the identification

$$
\operatorname{Aut}\left(S_{i}\right)^{m_{i}} \rtimes \Sigma_{m_{i}}=\prod_{k=1}^{i-1}\left\{\operatorname{id}_{S_{k}^{m_{k}}}\right\} \times\left(\operatorname{Aut}\left(S_{i}\right)^{m_{i}} \rtimes \Sigma_{m_{i}}\right) \times \prod_{k=i+1}^{n}\left\{\operatorname{id}_{S_{k}^{m_{k}}}\right\}
$$

we regard the group $\operatorname{Aut}\left(S_{i}\right)^{m_{i}} \rtimes \Sigma_{m_{i}}$ as a subgroup of $\operatorname{Aut}(M)$.

In this section, we prove the following

Theorem 4.1 (a) $\omega_{M} \simeq \mathcal{O}_{M}, H^{0}\left(M, T_{M}\right)=0, H^{1}\left(M, \mathcal{O}_{M}\right)=0$ and $\operatorname{Pic}(M)=$ $\mathrm{NS}(M)$ under the first Chern class map.

(b) Under the identification $\mathrm{NS}\left(S_{i j}\right)=p_{i j}^{*} \mathrm{NS}\left(S_{i j}\right)$ made above,

$$
\operatorname{Pic}(M)=\mathrm{NS}(M)=\prod_{i=1}^{n} \prod_{j=1}^{m_{i}} \mathrm{NS}\left(S_{i j}\right)
$$

(c) Under the identification (b),

$$
\overline{\operatorname{Mov}}(M)=\overline{\operatorname{Amp}}(M)=\prod_{i=1}^{n} \prod_{j=1}^{m_{i}} \overline{\operatorname{Amp}}\left(S_{i j}\right)
$$


(d) Under the inclusion $\operatorname{Aut}\left(S_{i}\right)^{m_{i}} \rtimes \Sigma_{m_{i}} \subset \operatorname{Aut}(M)$ explained above,

$$
\operatorname{Bir}(M)=\operatorname{Aut}(M)=\prod_{i=1}^{n}\left(\operatorname{Aut}\left(S_{i}\right)^{m_{i}} \rtimes \Sigma_{m_{i}}\right) .
$$

Proof The first assertion of (a) follows from the fact that $\omega_{S_{i}} \simeq \mathcal{O}_{S_{i}}$. The second (resp. the third) assertion of (a) follows from the fact that $h^{0}\left(T_{S_{i}}\right)=0\left(\operatorname{resp} . h^{1}\left(\mathcal{O}_{S_{i}}\right)=0\right)$ and the Künneth formula. The last assertion then follows from the exponential sequence. have

Let us prove (b). The first equality of (b) follows from (a). As $H^{1}\left(S_{i j}, \mathbb{Z}\right)=0$, we

$$
H^{2}(M, \mathbb{Z})=\prod_{i=1}^{n} \prod_{j=1}^{m_{i}} p_{i j}^{*} H^{2}\left(S_{i j}, \mathbb{Z}\right), \quad H^{1,1}(M, \mathbb{C})=\prod_{i=1}^{n} \prod_{j=1}^{m_{i}} p_{i j}^{*} H^{1,1}\left(S_{i j}, \mathbb{C}\right)
$$

by the Künneth formula. Thus the second equality follows from the Lefschetz $(1,1)$ theorem.

Let us prove (c). Choose points $Q_{i j} \in S_{i j}, 1 \leq i \leq n, 1 \leq j \leq m_{i}$. Let

$$
\iota_{i j}: S_{i j} \rightarrow M
$$

be the inclusion defined by

$$
x \mapsto\left(Q_{11}, Q_{12}, \ldots, Q_{i(j-1)}, x, Q_{i(j+1)}, \ldots, Q_{n m_{n}}\right)
$$

Note that $p_{i j} \circ \iota_{i j}=\operatorname{id}_{S_{i j}}$. Let $h \in \mathrm{NS}(M)$. Under the equality of the Néron-Severi groups in (b), we write

$$
h=\left(h_{11}, h_{12}, \ldots, h_{i j}, \ldots, h_{n m_{n}}\right) .
$$

Here $h_{i j} \in \mathrm{NS}\left(S_{i j}\right)$. Then $h=\sum_{i, j} p_{i j}^{*} h_{i j}$ and $h_{i j}=\iota_{i j}^{*} h$. As the pullback of the nef class is nef, the last equality in (c) follows from these two equalities.

By the last equality in (c), every projective birational contraction of $M$ is of the form

$$
\mu_{11} \times \mu_{12} \times \cdots \times \mu_{i j} \times \cdots \times \mu_{n m_{n}}: M \rightarrow V_{11} \times V_{12} \times \cdots \times V_{i j} \times \cdots \times V_{n m_{n}},
$$

where $\mu_{i j}: S_{i j} \rightarrow V_{i j}, 1 \leq i \leq n, 1 \leq j \leq m_{i}$, are birational projective contractions, in which some of $\mu_{i j}$ are possibly isomorphisms. Thus, $M$ has no small projective contraction. As $M$ is a smooth projective variety with $\omega_{M} \simeq \mathcal{O}_{M}$, the first equality of (c) then follows from [12, Theorem 5.7, Remark 5.9].

Let us prove (d). As $M$ has no small projective contraction, $M$ has no flop, either. Then, by the fundamental result due to Kawamata [14], it follows that $\operatorname{Bir}(M)=$ Aut $(M)$. Here we also used the fact that $M$ is a smooth projective variety with $\omega_{M} \simeq$ $\mathcal{O}_{M}$ in order to apply [14]. 
It remains to prove the last equality in (d). It is clear that the group in the right hand side is a subgroup of $\operatorname{Aut}(M)$. We shall prove the opposite inclusion by considering special fibrations on $M$. We proceed by the induction on $k=\sum m_{i j}$. The result is clear if $k=1$.

We call a surjective morphism $\varphi: M \rightarrow B$ a fibration if $B$ is a normal projective variety and the fibers of $f$ are connected. Note that a fibration is given by the morphism $\Phi_{|L|}$ associated to some complete, free linear system $|L|$. Indeed, $\varphi=\Phi_{\left|\varphi^{*} H\right|}$ for any very ample divisor $H$ on $B$, as $H^{0}(B, H) \simeq H^{0}\left(M, \varphi^{*} H\right)$ under $\varphi^{*}$, by the projection formula and the connectedness of fibers. Two fibrations $\varphi: M \rightarrow B$ and $(\varphi)^{\prime}: M \rightarrow B^{\prime}$ are called isomorphic if there is an isomorphism $\psi: B \rightarrow B^{\prime}$ such that $\psi \circ \varphi=(\varphi)^{\prime}$.

Let $S$ be a projective K3 surface. Then a fibration on $S$ is either an isomorphism, a birational contraction onto a K3 surface with Du Val singular points, a fibration $S \rightarrow \mathbb{P}^{1}$ whose general fiber is a smooth curve of genus one, or a surjective morphism to a point, Spec $\mathbb{C}$. These fibrations are given respectively by sufficiently positive multiples of an ample divisor, a non-ample nef, big divisor, a non-zero nef divisor with self-intersection number 0 and zero divisor. Therefore, by (c), a fibration $\varphi: M \rightarrow B$ is isomorphic to one of $p_{i j}: M \rightarrow S_{i j}$ if $B$ is a smooth K3 surface.

Let $f \in \operatorname{Aut}(M)$. Then $p_{11} \circ f: M \rightarrow S_{11}$ is a fibration. As $S_{11}$ is a smooth projective K3 surface, this fibration is isomorphic to one of $p_{i j}: M \rightarrow S_{i j}$. As $S_{i} \nsucceq S_{1}$ for $i \geq 2$ by our assumption, it follows that $i=1$. That is, there is $j, 1 \leq j \leq m_{1}$, such that $p_{11} \circ f: M \rightarrow S_{11}$ is isomorphic to $p_{1 j}: M \rightarrow S_{1 j}$. Choose an isomorphism

$$
g: S_{1 j} \rightarrow S_{11}
$$

such that

$$
g \circ p_{1 j}=p_{11} \circ f .
$$

Then $g \in \operatorname{Aut}\left(S_{1}\right)$, as $S_{1}=S_{1 j}$. Let $\sigma \in \operatorname{Aut}\left(S_{1}^{m_{1}}\right) \subset \operatorname{Aut}(M)$ be the automorphism defined by the permutation $(1, j) \in \Sigma_{m_{1}}$. By the definition, $p_{1 j} \circ \sigma=p_{11}$. Then

$$
p_{11} \circ(f \circ \sigma)=\left(p_{11} \circ f\right) \circ \sigma=\left(g \circ p_{1 j}\right) \circ \sigma=g \circ\left(p_{1 j} \circ \sigma\right)=g \circ p_{11} .
$$

Hence $f \circ \sigma \in \operatorname{Aut}(M)$ and $g \in \operatorname{Aut}\left(S_{1}\right)$ commute with the projection $p_{11}$. This means that $f \circ \sigma$ is of the form

$$
M=S_{11} \times N \ni(x, y) \mapsto\left(g(x), h_{x}(y)\right) \in S_{11} \times N=M .
$$

Here

$$
N=\prod_{j=2}^{m_{1}} S_{1 j} \times \prod_{i=2}^{n} \prod_{j=1}^{m_{j}} S_{i j}
$$


$x \in S_{11}$ and $y \in N$. Then the correspondence $x \mapsto h_{x}(\cdot)$ defines an analytic morphism

$$
\tau: S_{11} \rightarrow \operatorname{Aut}(N), \quad x \mapsto h_{x}(\cdot)
$$

As $H^{0}\left(N, T_{N}\right)=0$ by (a), $\operatorname{Aut}(N)$ is a discrete group. Hence $\tau$ is a constant map. This means that $f \circ \sigma$ is of the form

$$
M=S_{11} \times N \ni(x, y) \mapsto(g(x), h(y)) \in S_{11} \times N=M,
$$

for some automorphism $h \in \operatorname{Aut}(N)$, being independent of $x \in S_{11}$. Now, we can apply the assumption of the induction for $N$ and $h \in \operatorname{Aut}(N)$ and obtain the desired inclusion.

\section{An application for MDS and proof of Theorem 1.2 (b)}

In this section we shall prove Theorem 1.2(b). We prove it in a slightly generalized form as in Theorem 5.2 below.

We first recall the notion of MDS from [10]. Let $V$ be a normal $\mathbb{Q}$-factorial projective variety. A divisor $D$ on $V$ is called semi-ample if $|m D|$ is free for some positive integer $m$. We call a birational map $f: V \rightarrow V^{\prime}$ a small $\mathbb{Q}$-factorial modification if $V^{\prime}$ is a normal $\mathbb{Q}$-factorial projective variety and $f$ is isomorphic in codimension one, i.e., there are Zariski closed subsets $B \subset V$ and $B^{\prime} \subset V^{\prime}$ of codimension $\geq 2$, possibly empty, such that

$$
f \mid V \backslash B: V \backslash B \rightarrow V^{\prime} \backslash B^{\prime}
$$

is an isomorphism.

Definition 5.1 Let $V$ be a projective variety. $V$ is called an MDS if the following three conditions are satisfied:

(a) $V$ is normal $\mathbb{Q}$-factorial and $\operatorname{Pic}(V) \otimes \mathbb{Q}=\mathrm{NS}(V) \otimes \mathbb{Q}$ under the cycle map.

(b) The nef cone $\overline{\operatorname{Amp}}(V)$ is generated by finitely many semi-ample divisor classes as a convex cone.

(c) There are finitely many small $\mathbb{Q}$-factorial modifications $f_{i}: V \rightarrow V_{i}, 1 \leq i \leq n$, possibly $n=1$, such that $V_{i}$ satisfy (a) and (b) and

$$
\operatorname{Mov}(V)=\bigcup_{i=1}^{n} f_{i}^{*} \overline{\operatorname{Amp}}\left(V_{i}\right)
$$

The next theorem is the main result of this section.

Theorem 5.2 Let $S$ be a K3 surface such that $|\operatorname{Aut}(S)|<\infty$ and $\left|\operatorname{Aut}\left(S^{[n]}\right)\right|=\infty$. Then $S^{[n]}$ is not an MDS but $S^{(n)}$ is an MDS. In particular, the Hilbert-Chow morphism

$$
\nu: S^{[n]} \rightarrow S^{(n)}
$$


is a crepant projective resolution, which is also extremal in the sense of log minimal model program, but does not preserve MDS property.

Proof The fact that $v$ is a projective crepant extremal resolution is proved by Beauville [5, Proposition 5] based on a result of Forgaty. Then the proof follows from Claim 5.3, Proposition 5.4 and Claim 5.5 below.

Claim 5.3 $S^{[n]}$ is not an MDS. More strongly, $\overline{\operatorname{Amp}}\left(S^{[n]}\right)$ is not a finite rational polyhedral cone.

Proof We give a proof by contradiction, i.e., assuming to the contrary that $\overline{\operatorname{Amp}}\left(S^{[n]}\right)$ is a finite rational polyhedral cone, we prove $\left|\operatorname{Aut}\left(S^{[n]}\right)\right|<\infty$.

By the assumption, there are only finitely many 1-dimensional rays of the boundary of $\overline{\operatorname{Amp}}\left(S^{[n]}\right)$. We denote all such rays by $L_{k}, 1 \leq k \leq m$. Choose then the primitive integral generator $e_{k}$ of $L_{k}$. This is possible, as $L_{k}$ is defined over $\mathbb{Q}$, and $e_{k}$ is unique in each $L_{k}$. Consider the class

$$
H=\sum_{k=1}^{m} e_{k} \in \mathrm{NS}\left(S^{[n]}\right) \simeq \operatorname{Pic}\left(S^{[n]}\right) .
$$

As $\overline{\operatorname{Amp}}\left(S^{[n]}\right)$ is generated by $e_{k}, 1 \leq k \leq m$, as a convex cone and each $e_{k}$ is nef, it follows from Kleiman's criterion that $H$ is an ample class. By the definition, the set $\left\{e_{k}\right\}_{k=1}^{m}$ is stable under $\operatorname{Aut}\left(S^{[n]}\right)$. Hence $H$ is invariant under $\operatorname{Aut}\left(S^{[n]}\right)$ as well. Hence $\mid$ Aut $\left(S^{[n]}\right) \mid<\infty$ by [19, Proposition 2.4]. In order to apply [19, Proposition 2.4], we also used the fact that $H^{0}\left(S^{[n]}, T_{S^{[n]}}\right)=0$.

Let $S_{k}, 1 \leq k \leq n$, be projective K3 surfaces, possibly some of them are isomorphic, such that $\left|\operatorname{Aut}\left(S_{k}\right)\right|<\infty$ for each $k$. Set

$$
M=S_{1} \times S_{2} \times \cdots \times S_{n} .
$$

Proposition 5.4 $M$ is an $M D S$. More strongly, $M$ satisfies that $\operatorname{Pic}(M) \simeq \operatorname{NS}(M)$, $\overline{\operatorname{Mov}}(M)=\overline{\operatorname{Amp}}(M), \overline{\operatorname{Amp}}(M)$ is a finite rational polyhedral cone and any nef divisor of $M$ is semi-ample.

Proof By Theorem 4.1, $\operatorname{Pic}(M) \simeq \operatorname{NS}(M), \overline{\operatorname{Mov}}(M)=\overline{\operatorname{Amp}}(M)$.

By $\left|\operatorname{Aut}\left(S_{k}\right)\right|<\infty$, the nef cone $\overline{\operatorname{Amp}}\left(S_{k}\right)$ is a finite rational polyhedral cone. This follows from the solution of the cone conjecture for projective K3 surfaces ([22, Lemma 2.4], [13, Theorem 2.1]; see also [23, Theorem 3.3, Corollary 5.1], [1, Theorem 2.7] for closely related results). Note that every nef divisor $D$ on a projective $\mathrm{K} 3$ surface $S$ is effective. Indeed, this follows from the Riemann-Roch formula. As $\omega_{S} \simeq \mathcal{O}_{S}$, every nef divisor on $S$ is then semi-ample by the log abundance theorem in dimension 2 [9].

Hence, by Theorem 4.1, $\overline{\operatorname{Amp}}(M)$ is a finite rational polyhedral cone and every nef divisor on $M$ is semi-ample.

Claim $5.5 S^{(n)}$ is an MDS. 
Proof By Proposition 5.4, $S^{n}$ is an MDS. We have a surjective morphism

$$
S^{n} \rightarrow S^{(n)}, \quad\left(P_{1}, P_{2}, \ldots, P_{n}\right) \mapsto P_{1}+P_{2}+\cdots+P_{n} .
$$

Note that $S^{(n)}=S^{n} / \Sigma_{n}$ is a normal Q-factorial projective variety. As $S^{n}$ is an MDS by Proposition 5.4, so is $S^{(n)}$ by a fundamental result of Okawa [20, Theorem 1.1] (one may also apply [2, Theorem 1.1] in our situation).

Theorem 1.2(b) now follows from Theorem 5.2 and Theorem 1.2(a).

Acknowledgments The author expresses his best thanks to Doctor Malte Wandel for positing the question and to Professor Shinnosuke Okawa for discussions at the conference "Arithmetic and Algebraic Geometry" held at University of Tokyo, January 2014, from which this note grew up. Sincere thanks also go to Professors Alice Garbagnati, Fabrizio Catanese, Hélène Esnault, Brendan Hassett, Yujiro Kawamata, Thomas Peternell and Alessandra Sarti for valuable suggestions, discussions and encouragement, and the two referees for careful reading and showing a simpler proof of Claim 3.8. The author also expresses his thanks to Professors Toshiyuki Katsura, Iku Nakamura and Tomohide Terasoma for invitation to the above mentioned conference. The note is dedicated to Professor Fabrizio Catanese on the occasion of his sixty-fifth birthday, from whom the author has learned much about mathematics since 1993.

\section{References}

1. Artebani, M., Hausen, J., Laface, A.: On Cox rings of K3 surfaces. Compos. Math. 146(4), 964-998 (2010)

2. Bäker, H.: Good quotients of Mori dream spaces. Proc. Amer. Math. Soc. 139(9), 3135-3139 (2011)

3. Barth, W.P., Hulek, K., Peters, C.A.M., Van de Ven, A.: Compact Complex Surfaces. Ergebnisse der Mathematik und ihrer Grenzgebiete. A Series of Modern Surveys in Mathematics, vol. 4. Springer, Berlin (2004)

4. Beauville, A.: Some Remarks on Kähler Manifolds with $c_{1}=0$. In: Ueno, K. (ed.) Classification of Algebraic and Analytic Manifolds (Katata, 1982). Progress in Mathematics, vol. 39, pp. 1-26. Birkhäuser, Boston (1983)

5. Beauville, A.: Variétés Kähleriennes dont la première classe de Chern est nulle. J. Differential Geom. 18(4), 755-782 (1984)

6. Debarre, O.: Un contre-exemple au théorème de Torelli pour les variétés symplectiques irréductibles. C. R. Acad. Sci. Paris Sér. I Math. 299(14), 681-684 (1984)

7. Dolgachev, I.V.: Mirror symmetry for lattice polarized K3 surfaces. J. Math. Sci. 81(3), 2599-2630 (1996)

8. Fujiki, A.: On primitively symplectic compact Kähler $V$-manifolds of dimension four. In: Ueno, K. (ed.) Classification of Algebraic and Analytic Manifolds (Katata, 1982). Progress in Mathematics, vol. 39, pp. 71-250. Birkhäuser, Boston (1983)

9. Fujita, T.: Fractionally logarithmic canonical rings of algebraic surfaces. J. Fac. Sci. Univ. Tokyo Sect. IA Math. 30(3), 685-696 (1984)

10. Hu, Y., Keel, S.: Mori dream spaces and GIT. Michigan Math. J. 48, 331-348 (2000)

11. Kawamata, Y.: The cone of curves of algebraic varieties. Ann. Math. 119(3), 603-633 (1984)

12. Kawamata, Y.: Crepant blowing-up of 3-dimensional canonical singularities and its application to degenerations of surfaces. Ann. Math. 127(1), 93-163 (1988)

13. Kawamata, Y.: On the cone of divisors of Calabi-Yau fiber spaces. Internat. J. Math. 8(5), 665-687 (1997)

14. Kawamata, Y.: Flops connect minimal models. Publ. Res. Inst. Math. Sci. 44(2), 419-423 (2008)

15. Madonna, C., Nikulin, V.V.: On a classical correspondence between K3 surfaces. Proc. Steklov Inst. Math. 241, 120-153 (2003)

16. Markman, E., Yoshioka, K.: A proof of the Kawamata-Morrison Cone Conjecture for holomorphic symplectic varieties of $K 3^{[n]}$ or generalized Kummer deformation type (2014). arXiv:1402.2049

17. Morrison, D.R.: On K3 surfaces with large Picard number. Invent. Math. 75(1), 105-121 (1984) 
18. O’Grady, K.G.: Involutions and linear systems on holomorphic symplectic manifolds. Geom. Funct. Anal. 15(6), 1223-1274 (2005)

19. Oguiso, K.: Automorphism groups of Calabi-Yau manifolds of Picard number 2. J. Algebraic Geom. 23(4), 775-795 (2014)

20. Okawa, S.: On images of Mori dream spaces. Math. Ann. (2015). doi:10.1007/s00208-015-1245-5

21. Saint-Donat, B.: Projective models of $K-3$ surfaces. Amer. J. Math. 96(4), 602-639 (1974)

22. Sterk, H.: Finiteness results for algebraic K3 surfaces. Math. Z. 189(4), 507-513 (1985)

23. Totaro, B.: The cone conjecture for Calabi-Yau pairs in dimension 2. Duke Math. J. 154(2), 241-263 (2010) 